International Mathematical Forum, Vol. 9, 2014, no. 7, 335 - 353

HIKARI Ltd, www.m-hikari.com

http://dx.doi.org/10.12988/imf.2014.311205

\title{
Some Bitopological Properties via Grills
}

\author{
Sobhy Ahmed El-Sheikh \\ Mathematics Department, Faculty of Education \\ Ain Shams University, Cairo, Egypt
}

Copyright (c) 2014 Sobhy Ahmed El-Sheikh. This is an open access article distributed under the Creative Commons Attribution License, which permits unrestricted use, distribution, and reproduction in any medium, provided the original work is properly cited.

\begin{abstract}
In this paper we generate a bitopological space from the old one by using the notion of a grill and study the relation between these spaces. Given a bitopological space $\left(X, \tau_{1}, \tau_{2}\right)$ (bts, for short) and a grill $\mathcal{G}$ on $X$, we introduce a new local function $\Phi_{12}(A)=\left\{x \in X: O_{x} \cap A \in \mathcal{G} \forall O_{x} \in\right.$ $\left.\tau_{12}(x)\right\}$, where $\tau_{12}=\left\{U_{1} \cup U_{2}: U_{i} \in \tau_{i}, i=1,2\right\}$ is a supra topology [7] generated by $\tau_{1}$ and $\tau_{2},\left(X, \tau_{12}\right)$ is a supra topological space associate to the bts $\left(X, \tau_{1}, \tau_{2}\right)$. We show that $\Phi_{12}(A)=\Phi_{1}(A) \cap \Phi_{2}(A)$, where $\Phi_{i}(A): P(X) \longrightarrow P(X),(i=1,2)$, the given local functions associated to the spaces $\left(X, \tau_{i}\right)$. We show that the operator $c l_{12}^{*}(A)=A \cup \Phi_{12}(A)$ is a supra closure operator $[6,8]$ and then induces a supra topology $\tau_{12}^{*}$ which is finer than $\tau_{12}, \tau_{12}^{*}$ is not a topology in general. The properties and the relations between the spaces $\left(X, \tau_{12}\right),\left(X, \tau_{12}^{*}\right)$ and $\left(X, \tau_{1}^{*}, \tau_{2}^{*}\right)$ have investigated.
\end{abstract}

Mathematics Subject Classification: 54A05, 54A10, 54E55

Keywords: Bitopological space, grill, supra topology, connected, P.Hausdorff, P.regular, ideals, co-countable topology, closure operator, P.open, P.closed

\section{Introduction}

The idea of a grill on a topological space was first introduced by Choquet [4] in 1947. It is observed that from literature that the concept of grills is a powerful supporting tool, like nets and filters, in dealing with many a topological concept quite effectively. For instance proximity spaces, closure spaces and the theory of compactifications and similar other extensions problems are seen to have 
been tackled excellently by sheer use of grills (see $[3,2,12,14]$ for details). In this paper, given a bts $\left(X, \tau_{1}, \tau_{2}\right)$ and its associated supra topological space $\left(X, \tau_{12}\right)[6]$. Also, let $\mathcal{G}$ be a grill on a space $X$, we introduce a new local function, $\Phi_{12}: P(X) \longrightarrow P(X)$ and show that $\Phi_{12}(A)=\Phi_{1}(A) \cap \Phi_{2}(A)$. By making use of this function, we generate a family $\tau_{12}^{*}$ which is a supra topology and may be not a topology in general. The family $\tau_{12}^{*}$ is finer than $\tau_{1}, \tau_{2}$ and $\tau_{12}$. The properties of the operator $\Phi_{12}$ have obtained. Also, we investigate the relations between $\tau_{1}, \tau_{2}$ and $\tau_{12}$. We show that the operator $c l_{12}^{*}(A)=A \cup \Phi_{12}(A)$ is a supra closure operator and this operator induces the family $\tau_{12}^{*}$. This paper contains 5 sections. Section 2 is a Preliminary section. Section 3 concerns with the notion of the local function $\Phi_{12}$ and some of its properties. Section 4 devoted to more properties of the bts's and its relation with the grill. In section 5 , the suitability between the family $\tau_{12}$ and the grill $\mathcal{G}$ have obtained. Finally, some topological concepts related to such notion have given.

In what follows, by a space $X$, we shall mean a bts $\left(X, \tau_{1}, \tau_{2}\right)\left(\tau_{i}-c l\right.$ or $\left.\bar{A}^{i}\right)$ and $\left(\tau_{i}-i n t\right.$ or $\left.A^{i o}\right), i=1,2$, respectively, denote the $\tau_{i}$-closure and $\tau_{i}$-interior of $A$ in $X$. Also, the power set of $X$ will be denoted by $P(X)$ and $A^{\prime}$ or $X \backslash A$ will stand for the complement of $A$. A collection $\mathcal{G}$ of a nonempty subsets of a space $X$ is called a grill [14] on $X$, if it satisfies the following conditions:

1. $\emptyset \notin \mathcal{G}$,

2. $A \in \mathcal{G}$ and $A \subseteq B \Rightarrow B \in \mathcal{G}$, and

3. $A \cup B \in \mathcal{G} \Rightarrow A \in \mathcal{G}$ or $B \in \mathcal{G}$.

For any $x \in X$, we shall let $\tau_{i}(x)$ (resp. $\tau_{12}(x)$ ) to denote the collection of all $\tau_{i}\left(\tau_{12^{-}}\right)$open nbd of $x, i=1,2$.

\section{Preliminaries}

This section contains the notions which will be needed in the sequel, for more information see $[4,12,14]$.

Definition 2.1. Let $(X, \tau)$ be a topological space and $\mathcal{G}$ be a grill on $X$. We define a mapping $\Phi: P(X) \longrightarrow P(X)$, denoted by $\Phi_{\mathcal{G}}(A, \tau)$ or simply $\Phi(A)$, and defined by $\Phi_{\mathcal{G}}(A)=\left\{x \in X: O_{x} \cap A \in \mathcal{G} \forall O_{x} \in \tau(x)\right\}, \forall A \in P(X)$.

Proposition 2.1. Let $(X, \tau)$ be a topological space. Then

1. If $\mathcal{G}$ is any grill on $X$, then $\Phi$ is an increasing function in the sense that $A \subseteq B \Rightarrow \Phi(A) \subseteq \Phi(B)$ and if $\mathcal{G}_{1}, \mathcal{G}_{2}$ are two grills on $X$ with $\mathcal{G}_{1} \subseteq \mathcal{G}_{2}$, then $\Phi\left(\mathcal{G}_{1}\right) \subseteq \Phi\left(\mathcal{G}_{2}\right) \quad \forall A \subseteq X$. 
2. For any grill $\mathcal{G}$ on $X$ and any $A \subseteq X$ if $A \notin \mathcal{G}$, then $\Phi(A)=\emptyset$.

Proposition 2.2. Let $(X, \tau)$ be a topological space and $\mathcal{G}$ be a grill on $X$. Then for all $A, B \subseteq X$,

1. $\Phi(A \cup B)=\Phi(A) \cup \Phi(B)$.

2. $\Phi(\phi(A)) \subseteq \Phi(A)=\operatorname{cl}(\Phi(A)) \subseteq \operatorname{cl}(A)$.

If $\mathcal{G}$ is a grill on $X$. Define a mapping $c l^{*}: P(X) \longrightarrow P(X)$ by $\mathrm{cl}^{*}(A)=$ $A \cup \Phi(A) \forall A \subseteq X$. Then we have:

Theorem 2.1. The above map $\mathrm{cl}^{*}$ satisfies Kuratowski's closure axioms.

Definition 2.2. Corresponding to a grill $\mathcal{G}$ on a topological space $(X, \tau)$, there exists a unique topology $\tau_{\mathcal{G}}($ say) on $X$ given by:

$$
\tau_{\mathcal{G}}=\left\{U \subseteq X: c l^{*}\left(U^{\prime}\right)=U^{\prime}\right\}
$$

where for any $A \subseteq X, c l^{*}(A)=A \cup \Phi(A)=\tau_{\mathcal{G}}-c l(A), U^{\prime}$ is the complement of $U$.

Theorem 2.2. 1 . If $\mathcal{G}_{1}$ and $\mathcal{G}_{2}$ are two grills on a space $X$ with $\mathcal{G}_{1} \subseteq \mathcal{G}_{2}$, then $\tau_{\mathcal{G}_{2}} \subseteq \tau_{\mathcal{G}_{1}}$.

2. If $\mathcal{G}$ is a grill on a space $X$ and $B \notin \mathcal{G}$, then $B$ is closed in $\left(X, \tau_{\mathcal{G}}\right)$.

3. For any subset $A$ of a apace $X$ and any grill $\mathcal{G}$ on $X, \Phi(A)$ is $\tau_{\mathcal{G}}$-closed.

Definition 2.3. [9]. A bts is a triple $\left(X, \tau_{1}, \tau_{2}\right)$ where $\tau_{1}$ and $\tau_{2}$ are arbitrary topologies on $X$.

Definition 2.4. [5]. Let $\left(X, \tau_{1}, \tau_{2}\right)$ be a bts. Then $A \subseteq X$ is said to be pairwise open (P.open, for short) if $A=U_{1} \cup U_{2}, U_{i} \in \tau_{i},(i=1,2)$. A set $A$ is P.closed if its complement $A^{\prime}$ is P.open.

Note that the notion of P.open sets as well as P.closed sets has studied in [7, 6] under the name of $\tau_{12}$-open and $\tau_{12}$-closed.

Definition 2.5. [1]. A family $\eta \subseteq P(X)$ is said to be a supra topology on $X$ if $\eta$ contains $X, \emptyset$ and $\eta$ closed under arbitrary union. The elements of $\eta$ are supraopen sets and their complements are said to be supraclosed sets.

Proposition 2.3. [6, 5] Let $\left(X, \tau_{1}, \tau_{2}\right)$ be a bts. The family of all P.open subsets of $X$, denoted by

$$
\tau_{12}=\left\{U_{1} \cup U_{2}: U_{i} \in \tau_{i}, i=1,2\right\}
$$

is a supra topology on $X$ and $\left(X, \tau_{12}\right)$ is the supra topological space associated to the bts $\left(X, \tau_{1}, \tau_{2}\right)$. 
Definition 2.6. [8]. An operator $C: P(X) \longrightarrow P(X)$ is a supra closure closure operator if it satisfies the following conditions for all $A, B \subseteq X$.

Sc1 $C(\emptyset)=\emptyset$.

Sc2 $A \subseteq C(A)$.

Sc3 $C(A \cup B) \supseteq C(A) \cup C(B)$.

$S c_{4} C(C(A))=C(A)$.

Proposition 2.4. [7, 6]. Let $\left(X, \tau_{1}, \tau_{2}\right)$ be a bts. The operator cl $l_{12}: P(X) \longrightarrow$ $P(X)$ defined by $c_{12}(A)=\bar{A}^{1} \cap \bar{A}^{2}$ is a supra closure operator s.t $\tau_{12}=\{A \subseteq$ $\left.X: \operatorname{cl}_{12}\left(A^{\prime}\right)=A^{\prime}\right\}$.

Proposition 2.5. [6]. Let $\left(X, \tau_{1}, \tau_{2}\right)$ be a bts. The operator int I $_{12}: P(X) \longrightarrow$ $P(X)$ defined by $\operatorname{int}_{12}(A)=A^{o 1} \cup A^{o 2}$ is a supra interior operator s.t $\tau_{12}=$ $\left\{A \subseteq X: \operatorname{int}_{12}(A)=A\right\}$.

Now, we prove the following two proposition.

Proposition 2.6. Let $\left(X, \tau_{1}, \tau_{2}\right)$ be a bts and $A \subseteq X$. Then

1. $\tau_{1}, \tau_{2} \subseteq \tau_{12}$.

2. $\operatorname{cl}_{12}(A)=X \backslash \operatorname{int}_{12}(X \backslash A)$.

3. $\operatorname{int}_{12}(A)=X \backslash \operatorname{cl}_{12}(X \backslash A)$.

4. $A$ is P.open $\Leftrightarrow A=\operatorname{int}_{12}(A)$.

5. $A$ is P.closed $\Leftrightarrow A=\operatorname{cl}_{12}(A)$.

\section{Proof.}

Part (1) follows by definition of $\tau_{12}$. we prove the parts 2 and 4 . The proof of the others are similar.

(2)

$c_{12}(A)=\bar{A}^{1} \cap \bar{A}^{2}=X \backslash(X \backslash A)^{o 1} \cap X \backslash(X \backslash A)^{o 2}=X \backslash\left((X \backslash A)^{o 1} \cup(X \backslash A)^{o 2}\right)=$ $X \backslash\left(\operatorname{int}_{12}(X \backslash A)\right)$.

(4) Let $A$ be P.open. Then $A=U_{1} \cup U_{2}, U_{i} \in \tau_{i}(i=1,2)$. It follows that $\operatorname{int}_{12}(A)=\operatorname{int}_{12}\left(U_{1} \cup U_{2}\right)=\left(U_{1} \cup U_{2}\right)^{o 1} \cup\left(U_{1} \cup U_{2}\right)^{o 2} \supseteq U_{1}^{o 1} \cup U_{2}^{o 1} \cup U_{1}^{o 2} \cup$ $U_{2}^{o 2}=U_{1} \cup U_{2}^{o 1} \cup U_{1}^{o 2} \cup U_{2} \supseteq U_{1} \cup U_{2}=A$. Hence $A \subseteq \operatorname{int}_{12}(A)$, but clearly $\operatorname{int}_{12}(A) \subseteq A$. So $A=\operatorname{int}_{12}(A)$. Conversely, let $A=\operatorname{int}_{12}(A)$. Then $A=A^{o 1} \cup A^{o 2}, A^{o i} \in \tau_{i}(i=1,2)$ and consequently $A$ is P.open.

Proposition 2.7. Let $\left(X, \tau_{1}, \tau_{2}\right)$ be a bts and $A \subseteq X$. Then $x \in c l_{12}(A) \Leftrightarrow$ $\forall O_{x} \in \tau_{12}, O_{x} \cap A \neq \phi$. 


\section{Proof.}

Let $x \in \operatorname{cl}_{12}(A)$ and $O_{x} \cap A=\emptyset$ for some $O_{x} \in \tau_{12}(x)$. Then $O_{x}=O_{x}^{1} \cup O_{x}^{2}$, $O_{x}^{i} \in \tau_{i}(i=1,2)$. It follows that, $\left(O_{x}^{1} \cup O_{x}^{2}\right) \cap A=\emptyset \Rightarrow O_{x}^{1} \cap A=\emptyset$ and $O_{x}^{2} \cap A=\emptyset$. Now $x \in O_{x} \Rightarrow x \in O_{x}^{1}$ or $x \in O_{x}^{2}$. If $x \in O_{x}^{1}, O_{x}^{1} \cap A=\emptyset$, we have $x \notin \bar{A}^{1}$ and consequently $x \notin c l_{12}(A)$, which is a contradiction. Also, $x \in O_{x}^{2}$, $O_{x}^{2} \cap A=\emptyset$, gives $x \notin \bar{A}^{2} \Rightarrow x \notin c l_{12}(A)$, which is also a contradiction. Conversely, let $O_{x} \cap A \neq \emptyset \forall O_{x} \in \tau_{12}(x)$ and let $x \notin c l_{12}(A)$. Then $x \notin \bar{A}^{1}$ or $x \notin \bar{A}^{2}$. So, $x \notin \bar{A}^{1} \Rightarrow \exists O_{x} \in \tau_{1} \subseteq \tau_{12}$ s.t $O_{x} \cap A=\emptyset$. Also, $x \notin \bar{A}^{2} \Rightarrow \exists O_{x} \in \tau_{2} \subseteq \tau_{12}$ s.t $O_{x} \cap A=\emptyset$. In both cases we have a contradiction.

\section{Bitopological spaces and the operator $\Phi_{12}$}

In this section, we consider $\left(X, \tau_{1}, \tau_{2}\right)$ as a bts, and $\left(X, \tau_{12}\right)$ its associated supra topological space and $\mathcal{G}$ be a grill on $X$.

Definition 3.1. Let $\left(X, \tau_{1}, \tau_{2}\right)$ be a bts, $\mathcal{G}$ be a grill on a space $X$ and $A \subseteq X$. Then the operator $\Phi_{12}: P(X) \rightarrow P(X)$ given by $\Phi_{12}(A)=\left\{x \in X: O_{x} \cap A \in\right.$ $\left.\mathcal{G} \forall O_{x} \in \tau_{12}(x)\right\}$ is a local function associated with $\mathcal{G}$.

Proposition 3.1. Let $\left(X, \tau_{1}, \tau_{2}\right)$ be a bts. Then:

(i) If $\mathcal{G}$ is any grill on $X$, then $\Phi_{12}$ is an increasing function, i.e. $A \subseteq B(\subseteq$ $X) \Rightarrow \Phi_{12}(A) \subseteq \Phi_{12}(B)$.

(ii) If $\mathcal{G}_{1}$ and $\mathcal{G}_{2}$ are two grills on $X$ with $\mathcal{G}_{1} \subseteq \mathcal{G}_{2}$, then $\Phi_{12}^{\mathcal{G}_{1}}(A) \subseteq \Phi_{12}^{\mathcal{G}_{2}}(A) \forall A \subseteq$ $X$.

(iii) For any grill $\mathcal{G}$ on $X$ and $A \subseteq X$, if $A \notin \mathcal{G}$, then $\Phi_{12}(A)=\emptyset$.

Proof. It follows from the definition of the local function $\Phi_{12}$.

Proposition 3.2. Let $\left(X, \tau_{1}, \tau_{2}\right)$ be a bts and $\mathcal{G}$ be a grill on $X$. Then for all $A, B \subseteq X$

(i) $\Phi_{12}(A \cup B) \supseteq \Phi_{12}(A) \cup \Phi_{12}(B)$,

(ii) $\Phi_{12}\left(\Phi_{12}(A)\right) \subseteq \Phi_{12}(A)=\operatorname{cl}_{12}\left(\Phi_{12}(A)\right) \subseteq c l_{12}(A)$.

\section{Proof.}

(i) Since $A, B \subseteq A \cup B$, by Proposition 3.1 (i), $\Phi_{12}(A) \subseteq \Phi_{12}(A \cup B)$ and $\Phi_{12}(B) \subseteq \Phi_{12}(A \cup B)$. It follows that $\Phi_{12}(A) \cup \Phi_{12}(B) \subseteq \Phi_{12}(A \cup B)$.

(ii) To prove that $\Phi_{12}\left(\Phi_{12}(A)\right) \subseteq \Phi_{12}(A)$ let $x \in \Phi_{12}\left(\Phi_{12}(A)\right)$. Then $O_{x} \cap$ $\Phi_{12}(A) \in \mathcal{G}, \forall O_{x} \in \tau_{12}(X)$. So, $O_{x} \cap \Phi_{12}(A) \neq \emptyset$ and consequently there exists 
$y \in O_{x} \cap \Phi_{12}(A)$. Then $y \in O_{x}$ and $y \in \Phi_{12}(A)$. Thus, $O_{y} \cap A \in \mathcal{G}$ for all $O_{y} \in \tau_{12}(y)$. Since $y \in O_{x}, O_{x} \cap A \in \mathcal{G}$, so $x \in \Phi_{12}(A)$ and therefore $\Phi_{12}\left(\Phi_{12}(A)\right) \subseteq \Phi_{12}(A)$.

Clearly, $\Phi_{12}(A) \subseteq \operatorname{cl}_{12}\left(\Phi_{12}(A)\right)$, so, we prove that $\mathrm{ll}_{12}\left(\Phi_{12}(A)\right) \subseteq \Phi_{12}(A)$. Thus, let $x \in \operatorname{cl}_{12}\left(\Phi_{12}(A)\right)$. Then $\forall O_{x} \in \tau_{12}(x) ; O_{x} \cap \Phi_{12}(A) \neq \emptyset$. So, there exists $y \in O_{x} \cap \Phi_{12}(A)$. It follows that $y \in O_{x}$ and $y \in \Phi_{12}(A)$. So, for all $O_{y} \in \tau_{12}(y), O_{y} \cap A \in \mathcal{G}$. Hence $O_{x} \cap A \in \mathcal{G}$ and this yields $x \in \Phi_{12}(A)$. Finally, we have $\Phi_{12}(A) \supseteq c_{12}\left(\Phi_{12}(A)\right)$ and consequently $\Phi_{12}(A)=c l_{12}\left(\Phi_{12}(A)\right)$. Now to complete the proof of part (ii), we show that $\Phi_{12}(A) \subseteq \operatorname{cl}_{12}(A)$. So, let $x \notin c l_{12}(A)$. Then there exists $O_{x} \in \tau_{12}(x)$ such that $O_{x} \cap A=\emptyset$, then $x \notin \Phi_{12}(A)$ and consequently $\Phi_{12}(A) \subseteq c_{12}(A)$.

Remark 3.1. Let $\left(X, \tau_{1}, \tau_{2}\right)$ be a bts and $\mathcal{G}$ be a grill on $X$. Let $\left(X, \tau_{1}^{*}, \tau_{2}^{*}\right)$ be a bts induced by $\mathcal{G}$, where

$$
\begin{gathered}
\tau_{1}^{*}=\left\{A \subseteq X: c l_{1}^{*}(X \backslash A)=X \backslash A\right\}, \\
\tau_{2}^{*}=\left\{A \subseteq X: c l_{2}^{*}(X \backslash A)=X \backslash A\right\}, \\
c l_{i}^{*}(A)=A \cup \Phi_{i}(A)(i=1,2) \text { and } \\
\Phi_{i}(A)=\left\{x \in X: O_{x} \cap A \in \mathcal{G} \forall O_{x} \in \tau_{i}(x)\right\} .
\end{gathered}
$$

Also, note that $\tau_{i} \subseteq \tau_{i}^{*}$.

Lemma 3.1. Let $\left(X, \tau_{1}, \tau_{2}\right)$ be a bts and $\mathcal{G}$ be a grill on $X$. Let $\Phi_{12}: P(X) \rightarrow$ $P(X)$ be a local function. Then

$$
\Phi_{12}(A)=\Phi_{1}(A) \cap \Phi_{2}(A) \forall, A \subseteq X
$$

\section{Proof.}

Let $x \notin \Phi_{1}(A) \cap \Phi_{2}(A)$. Then $x \notin \Phi_{1}(A)$ or $x \notin \Phi_{2}(A)$. If $x \notin \Phi_{1}(A)$, then there exists $O_{x} \in \tau_{1} \subseteq \tau_{12}$ such that $O_{x} \cap A \notin \mathcal{G}$. Hence $x \notin \Phi_{12}(A)$. Similarly, if $x \notin \Phi_{2}(A)$, then there exists $O_{x} \in \tau_{2} \subseteq \tau_{12}$ such that $O_{x} \cap A \notin \mathcal{G}$. Hence $x \notin \Phi_{12}(A)$. So, in both cases, $\Phi_{12}(A) \subseteq \Phi_{1}(A) \cap \Phi_{2}(A)$. On the other hand, if $x \notin \Phi_{12}(A)$, then there exists $O_{x} \in \tau_{12}(x)$ such that $O_{x} \cap A \notin \mathcal{G}$. Now, $O_{x} \in$ $\tau_{12}(x) \Rightarrow O_{x}=O_{x}^{1} \cup O_{x}^{2}\left(O_{x}^{i} \in \tau_{i}, i=1,2\right) \Rightarrow\left(O_{x}^{1} \cup O_{x}^{2}\right) \cap A \notin \mathcal{G} \Rightarrow O_{x}^{i} \cap A \notin \mathcal{G}$ (since $\mathcal{G}$ is a grill). Now, $x \in O_{x} \Rightarrow x \in O_{x}^{1}$ or $x \in O_{x}^{2} \Rightarrow O_{x}^{1} \cap A \notin \mathcal{G}$ or $O_{x}^{2} \cap A \notin \mathcal{G} \Rightarrow x \notin \Phi_{1}(A)$ or $x \notin \Phi_{2}(A) \Rightarrow x \notin \Phi_{1}(A) \cap \Phi_{2}(A)$. Hence the result. 
The following theorem gives the properties of the local function $\Phi_{12}$ in terms of the local functions $\Phi_{1}$ and $\Phi_{2}$.

Theorem 3.1. Let $\left(X, \tau_{1}, \tau_{2}\right)$ be a bts and $\mathcal{G}$ be a grill on $X$. Then, the local function $\Phi_{12}(A)=\Phi_{1}(A) \cap \Phi_{2}(A)$ satisfies the following properties.

(i) $\Phi_{12}(\phi)=\phi$,

(ii) $A \subseteq B \Rightarrow \Phi_{12}(A) \subseteq \Phi_{12}(B)$,

(iii) $\Phi_{12}(A) \cup \Phi_{12}(B) \subseteq \Phi_{12}(A \cup B)$

(iv) $\Phi_{12}\left(\Phi_{12}(A)\right) \subseteq \Phi_{12}(A)=\operatorname{cl}_{12}\left(\Phi_{12}(A)\right) \subseteq c l_{12}(A)$.

\section{Proof.}

(i) $\Phi_{12}(\phi)=\Phi_{1}(\phi) \cap \Phi_{2}(\phi)=\phi$.

(ii) Let $A \subseteq B$. Then $\Phi_{12}(A)=\Phi_{1}(A) \cap \Phi_{2}(A) \subseteq \Phi_{1}(B) \cap \Phi_{2}(B)=\Phi_{12}(B)$

(by using the properties of $\Phi_{1}, \Phi_{2}$ ).

(iii) Follows from (ii).

(iv) $\Phi_{12}\left(\Phi_{12}(A)\right)=\Phi_{1}\left(\Phi_{12}(A)\right) \cap \Phi_{2}\left(\Phi_{12}(A)\right)$

$$
\begin{aligned}
& =\Phi_{1}\left(\Phi_{1}(A) \cap \Phi_{2}(A)\right) \cap \Phi_{2}\left(\Phi_{1}(A) \cap \Phi_{2}(A)\right) \\
& \subseteq \Phi_{1}\left(\Phi_{1}(A)\right) \cap \Phi_{1}\left(\Phi_{2}(A)\right) \cap \Phi_{2}\left(\Phi_{1}(A)\right) \cap \Phi_{2}\left(\Phi_{2}(A)\right. \\
& \subseteq \Phi_{1}(A) \cap \Phi_{1}\left(\Phi_{2}(A)\right) \cap \Phi_{2}(\Phi(A)) \cap \Phi_{2}(A) . \\
& \subseteq \Phi_{1}(A) \cap \Phi_{2}(A)=\Phi_{12}(A) .
\end{aligned}
$$

Hence $\Phi_{12}\left(\Phi_{12}(A)\right) \subseteq \Phi_{12}(A)$.

Clearly, $\Phi_{12}(A) \subseteq \operatorname{cl}_{12}\left(\Phi_{12}(A)\right)$.

On the other hand, $c_{12}\left(\Phi_{12}(A)\right)={\overline{\Phi_{12}(A)}}^{1} \cap{\overline{\Phi_{12}(A)}}^{2}$

$i=1,2)$

$$
\begin{aligned}
& ={\overline{\Phi_{1}(A) \cap \Phi_{2}(A)}}^{1} \cap{\overline{\Phi_{1}(A) \cap \Phi_{2}(A)}}^{2} \\
& \subseteq{\overline{\Phi_{1}(A)}}^{1} \cap{\overline{\Phi_{2}(A)}}^{1} \cap{\overline{\Phi_{1}(A)}}^{2} \cap{\overline{\Phi_{2}(A)}}^{2} \\
& =\Phi_{1}(A) \cap{\overline{\Phi_{2}(A)}}^{1} \cap{\overline{\Phi_{1}(A)}}^{2} \cap \Phi_{2}(A)\left(\text { since }{\overline{\Phi_{i}(A)}}^{i}=\Phi_{i}(A),\right.
\end{aligned}
$$

$$
=\Phi_{1}(A) \cap \Phi_{2}(A)=\Phi_{12}(A) \text {. Hence } \Phi_{12}(A)=\operatorname{cl}_{12}\left(\Phi_{12}(A)\right) .
$$

Finally, we show that $\Phi_{12}(A) \subseteq c l_{12}(A)$. Since, $\Phi_{12}(A)=\Phi_{1}(A) \cap \Phi_{2}(A) \subseteq$ $\bar{A}^{1} \cap \bar{A}^{2}=c_{12}(A)\left(\right.$ since $\left.\Phi_{i}(A) \subseteq \bar{A}^{i}, i=1,2\right)$.

If $\mathcal{G}$ is a grill on a space $\left(X, \tau_{1}, \tau_{2}\right)$. Define a mapping cl $l_{12}^{*}: P(X) \rightarrow P(X)$ by $c_{12}^{*}(A)=A \cup \Phi_{12}(A) \forall A \subseteq X$. Then we have the following theorem.

Theorem 3.2. The above map $\mathrm{cl}_{12}^{*}$ is a supra closure operator which induces the supra topology $\tau_{12}^{*}=\left\{A \subseteq X: c l_{12}^{*}(X \backslash A)=X \backslash A\right\}$.

Proof.

Let $c l_{12}^{*}(A)=A \cup \Phi_{12}(A)$. Then $(S C 1) c l_{12}^{*}(\phi)=\phi \cup \Phi_{12}(\phi)=\phi$.

(SC2) Clearly, $A \subseteq \mathrm{cl}_{12}^{*}(A)$

Note that if $A \subseteq B$, then $c_{12}^{*}(A)=A \cup \Phi_{12}(A) \subseteq B \cup \Phi_{12}(B)=c l_{12}^{*}(B)$, i.e. 
$A \subseteq B \Rightarrow c l_{12}^{*}(A) \subseteq c l_{12}^{*}(B)$.

(SC3) $c l_{12}^{*}(A) \cup c l_{12}^{*}(B) \subseteq c l_{12}^{*}(A \cup B)$ (follows from the above note).

(SC4) The proof follows by using the properties of $\Phi_{1}, \Phi_{2}$ and by using (SC2).

Hence $\mathrm{cl}_{12}^{*}$ is a supra closure operator.

it is easy to show that the family

$$
\tau_{12}^{*}=\left\{A \subseteq X: c l_{12}^{*}(X \backslash A)=X \backslash A\right\}
$$

is a supra topology on $X$ it is not a topology in general (see Example 3.1 below).

Definition 3.2. Corresponding to a grill $\mathcal{G}$ on a bts $\left(X, \tau_{1}, \tau_{2}\right)$ there exists a unique supra topology $\tau_{12}^{*}($ say) on $X$ given by

$$
\tau_{12}^{*}=\left\{U \subseteq X: c l_{12}^{*}(X \backslash U)=X \backslash U\right\}
$$

which is finer than $\tau_{12}$ and $c l_{12}^{*}(A)=A \cup \Phi_{12}(A)=\tau_{12}^{*}-\operatorname{cl}(A) \forall A \subseteq X$.

Theorem 3.3. Let $\left(X, \tau_{1}, \tau_{2}\right)$ be a bts, $\mathcal{G}$ be a grill on $X$ and $A \subseteq X$. Then

$$
c l_{12}^{*}(A)=A \cup \Phi_{12}(A)=c l_{1}^{*}(A) \cap c l_{2}^{*}(A) .
$$

Proof.

Since $c l_{12}^{*}(A)=A \cup \Phi_{12}(A)$, then

$c l_{12}^{*}(A)=A \cup\left(\Phi_{1}(A) \cap \Phi_{2}(A)\right)$

$=\left(A \cup \Phi_{1}(A)\right) \cap\left(A \cup \Phi_{2}(A)\right)$

$=c l_{1}^{*}(A) \cap c l_{2}^{*}(A)$.

Note that the above Theorem means that we can established the same supra topology from a bts $\left(X, \tau_{1}, \tau_{2}\right)$ by using two equivalent methods. The first follows from the local function $\Phi_{12}$ and the other by using the closure operators $c l_{1}^{*}, c l_{2}^{*}$ induced by the local functions $\Phi_{1}, \Phi_{2}$.

Theorem 3.4. Let $\left(X, \tau_{1}, \tau_{2}\right)$ be a bts, $\mathcal{G}$ be a grill on $X$. Let $\left(X, \tau_{1}{ }^{*}, \tau_{2}{ }^{*}\right)$ be a bts induced by $\mathcal{G}$ and the local functions $\Phi_{1}$ and $\Phi_{2}$. Then

$$
\tau_{12}^{*}=\left\{U_{1} \cup U_{2}: U_{i} \in \tau_{i}^{*}, i=1,2\right\}
$$

\section{Proof.}

Let $A \in \tau^{*}{ }_{12}$. Then $c_{12}^{*}(X \backslash A)=X \backslash A$

$\Rightarrow X \backslash A=c l_{1}^{*}(X \backslash A) \cap c l_{2}^{*}(X \backslash A)$

$\Rightarrow X \backslash A=X \backslash i n t_{1}^{*}(A) \cap X \backslash i n t_{2}^{*}(A)$

$\Rightarrow A=i n t_{1}^{*}(A) \cup i n t_{2}^{*}(A)=U_{1} \cup U_{2}, U_{i} \in \tau_{i}^{*}$.

Conversely, let $A==U_{1} \cup U_{2}, U_{i} \in \tau_{i}^{*}$. Then $c l_{12}^{*}(X \backslash A)=c l_{12}^{*}\left(X \backslash U_{1} \cap\right.$ $\left.X \backslash U_{2}\right)=c l_{1}^{*}\left(X \backslash U_{1} \cap X \backslash U_{2}\right) \cap c l_{2}^{*}\left(X \backslash U_{1} \cap X \backslash U_{2}\right) \subseteq c l_{1}^{*}\left(X \backslash U_{1}\right) \cap c l_{1}^{*}\left(X \backslash U_{2}\right) \cap$ $c l_{2}^{*}\left(X \backslash U_{1}\right) \cap c l_{2}^{*}\left(X \backslash U_{2}\right)=X \backslash U_{1} \cap c l_{1}^{*}\left(X \backslash U_{2}\right) \cap c l_{2}^{*}\left(X \backslash U_{1}\right) \cap X \backslash U_{2}=X \backslash U_{1} \cap$ $X \backslash U_{2}=X \backslash A$. But, $X \backslash A \subseteq c l_{12}^{*}(X \backslash A)$. Hence $c l_{12}^{*}(X \backslash A)=X \backslash A$ and consequently $A \in \tau_{12}^{*}$. 
Remark 3.2. Let $\left(X, \tau_{1}, \tau_{2}\right)$ be a bts, $\mathcal{G}$ be a grill on $X$. Then

(1) $\Phi_{12}(A \cup B) \neq \Phi_{12}(A) \cup \Phi_{12}(B)$ in general.

(2) $c l_{12}^{*}(A \cup B) \neq c l_{12}^{*}(A) \cup c l_{12}^{*}(B)$ in general.

(3) $\tau_{12}^{*}$ which induced by $\mathrm{cl}_{12}^{*}$ may be not a topology in general but it is a supra topology finer than $\tau_{1}, \tau_{2}$ and $\tau_{12}$.

(4) $\tau_{1} \cup \tau_{2} \subseteq \tau_{12} \subseteq \tau_{12}^{*}$.

Example 3.1. Let $X=\{a, b, c\}, \tau_{1}$ and $\tau_{2}$ be two topologies on $X$ such that $\tau_{1}=\{\emptyset, X,\{a, b\}\}$ and $\tau_{2}=\{\emptyset, X,\{a, c\}\}$. Let $\mathcal{G}=P(X) \backslash \emptyset$. Now, let $A=$ $\{b\}, B=\{c\}$, then $\tau_{12}=\{\emptyset, X,\{a, b\},\{a, c\}\}$ is a supra topology, since $\{a, b\} \cap$ $\{a, c\} \notin \tau_{12}$. Since $\Phi_{12}(\{b\})=\{b\}, \Phi_{12}(\{c\})=\{c\}$ and $\Phi_{12}(\{b, c\})=X$, then $\Phi_{12}(A \cup B) \neq \Phi_{12}(A) \cup \Phi_{12}(B)$ and consequently, $c l_{12}^{*}(A \cup B) \neq c l_{12}^{*}(A) \cup c l_{12}^{*}(B)$. Also, $\tau_{12}^{*}=\tau_{12}, \tau_{1}, \tau_{2} \subseteq \tau_{12}$ and $\tau_{12}^{*}$ is a supra topology.

Proposition 3.3. (a) Let $\mathcal{G}_{1}$ and $\mathcal{G}_{2}$ be two grills on a space $X$ with $\mathcal{G}_{1} \subseteq \mathcal{G}_{2}$. Then $\tau_{12}^{*}\left(\mathcal{G}_{2}\right) \subseteq \tau_{12}^{*}\left(\mathcal{G}_{1}\right)$

(b) If $\mathcal{G}$ is a grill on a space $\left(X, \tau_{1}, \tau_{2}\right)$ and $B \notin \mathcal{G}$, then $B$ is P.closed in $\left(X, \tau_{1}^{*}, \tau_{2}^{*}\right)$.

(c) For any subset $A$ of a space $X$ and any grill $\mathcal{G}$ on $X, \Phi_{12}(A)$ is P.closed in $\left(X, \tau_{1}^{*}, \tau_{2}^{*}\right)$ or it is $\tau_{12}^{*}$-closed.

\section{Proof.}

(a) Let $U \in \tau_{12}^{*}\left(\mathcal{G}_{2}\right)$. Then $X \backslash U=\tau_{12}^{*}\left(\mathcal{G}_{2}\right)-\operatorname{cl}(X \backslash U)=X \backslash U \cup \Phi_{12}^{\mathcal{G}_{2}}(X \backslash U)$

$\Rightarrow \Phi_{12}^{\mathcal{G}_{2}}(X \backslash U) \subseteq X \backslash U$

$\Rightarrow \Phi_{12}^{\mathcal{G}_{1}}(X \backslash U) \subseteq \Phi_{12}^{\mathcal{G}_{2}}(X \backslash U) \subseteq X \backslash U$ (by Proposition 3.1 (ii) )

$\Rightarrow X \backslash U=X \backslash U \cup \Phi_{12}^{\mathcal{G}_{1}}(X \backslash U)=\tau_{12}^{*}\left(\mathcal{G}_{1}\right)-\operatorname{cl}(X \backslash U)$

$\Rightarrow U \in \tau_{12}^{*}\left(\mathcal{G}_{1}\right)$, i.e. $\tau_{12}^{*}\left(\mathcal{G}_{2}\right) \subseteq \tau_{12}^{*}\left(\mathcal{G}_{1}\right)$.

(b) By Proposition 3.1(iii), $B \notin \mathcal{G} \Rightarrow \Phi_{12}(B)=\phi \Rightarrow c_{12}^{*}(B)=B \Rightarrow B$ is a $\tau_{12}^{*}$-closed or P.closed in $\left(X, \tau_{1}^{*}, \tau_{2}^{*}\right)$. Another proof, let $B \notin \mathcal{G}$. Then $B$ is $\tau_{1}^{*}$-closed and $\tau_{2}^{*}$-closed. So, $\Phi_{1}(B) \subseteq B$ and $\Phi_{2}(B) \subseteq B \Rightarrow \Phi_{12}(B)=$ $\Phi_{1}(B) \cap \Phi_{2}(B) \subseteq B \Rightarrow \Phi_{12}(B) \subseteq B$. Hence $B=B \cup \Phi_{12}(B)=c l_{12}^{*}(B) \Rightarrow B$ is P.closed in $\left(X, \tau_{1}^{*}, \tau_{2}^{*}\right)$.

(c) Since $c l_{12}^{*}\left(\Phi_{12}(A)\right)=\Phi_{12}(A) \cup \Phi_{12}\left(\Phi_{12}(A)\right) \Rightarrow c l_{12}^{*}\left(\Phi_{12}(A)\right)=\Phi_{12}(A)$ ( since $\left.\Phi_{12}\left(\Phi_{12}(A)\right) \subseteq \Phi_{12}(A)\right) \Rightarrow \tau_{12}^{*}-c l\left(\Phi_{12}(A)\right)=\Phi_{12}(A) \Rightarrow \Phi_{12}(A)$ is a $\tau_{12}^{*}$-closed or P.closed in $\left(X, \tau_{1}^{*}, \tau_{2}^{*}\right)$.

\section{More properties on bts's and grills}

Theorem 4.1. Let $\left(X, \tau_{1}, \tau_{2}\right)$ be a bts and $\mathcal{G}$ be a grill on $X$. Then 


$$
\beta\left(\mathcal{G}, \tau_{12}\right)=\left\{V \backslash A: V \in \tau_{12}, A \notin \mathcal{G}\right\}
$$

is an open base for $\tau_{12}^{*}$.

\section{Proof.}

Let $U \in \tau_{12}^{*}$ and $x \in U$. Then $X \backslash U$ is $P$-closed set so that $c l_{12}^{*}(X \backslash U)=$ $X \backslash U$ and hence, $X \backslash U=X \backslash U \cup \Phi_{12}(X \backslash U) \Rightarrow \Phi_{12}(X \backslash U) \subseteq X \backslash U$, then $x \notin$ $\Phi_{12}(X \backslash U)$. So $\exists V \in \tau_{12}(x)$ such that $V \cap X \backslash U \notin \mathcal{G}$. Let $A=V \cap X \backslash U$. Then $x \notin A$ and $A \notin \mathcal{G}$. Thus $x \in V \backslash A \subseteq V \backslash(V \cap X \backslash U)=U$, i.e. $x \in V \backslash A \subseteq U$, where $V \backslash A \in \beta\left(\mathcal{G}, \tau_{12}\right)$.

Note that $\beta\left(\mathcal{G}, \tau_{12}\right)$ may be not closed under finite intersection, since $\tau_{12}$ is allready not closed under finite intersection (since $\tau_{12}$ is a supra topology). Therefore, $\tau_{12}^{*}$ may be not a topology, so we only need the condition that every member of $\tau_{12}^{*}$ is a union of members of $\tau_{12}$.

Definition 4.1. Let $\left(X, \tau_{1}, \tau_{2}\right)$ be a bts and $\mathcal{G}$ be a grill on $X$. Then $\beta\left(\mathcal{G}, \tau_{12}\right)$ is a base for $\tau_{12}^{*}$ if $\forall U \in \tau_{12}^{*}, x \in U \exists V \backslash A \in \beta$ such that $x \in V \backslash A \subseteq U$.

Corollary 4.1. For any grill $\mathcal{G}$ on a bts $\left(X, \tau_{1}, \tau_{2}\right), \tau_{12} \subseteq \beta\left(\mathcal{G}, \tau_{12}\right) \subseteq \tau_{12}^{*}$.

Proof. Let $V \in \tau_{12}, \phi \notin \mathcal{G}$. Then $V=V \backslash \phi \in \beta\left(\mathcal{G}, \tau_{12}\right) \subseteq \tau_{12}^{*}$.

Example 4.1. Let $\left(X, \tau_{1}, \tau_{2}\right)$ be a bts. If $\mathcal{G}=P(X) \backslash\{\emptyset\}$. Then $\tau_{12}=\tau_{12}^{*}$. In fact, for any $\tau_{12}^{*}$-basic open set $V=U \backslash A, U \in \tau_{12}$ and $A \notin \mathcal{G}$, we have $A=\emptyset$, so that $V=U \in \tau_{12}, V=U \in \beta\left(\mathcal{G}, \tau_{12}\right)$ that is $\beta\left(\mathcal{G}, \tau_{12}\right) \subseteq \tau_{12} \subseteq \beta\left(\mathcal{G}, \tau_{12}\right)$. Hence $\tau_{12}=\beta\left(\mathcal{G}, \tau_{12}\right)=\tau_{12}^{*}$.

Theorem 4.2. Let $\left(X, \tau_{1}, \tau_{2}\right)$ be a bts and $\mathcal{G}$ be a grill on $X$. If $\tau_{12}$ is a topology and $U \in \tau_{12}$, then $U \cap \Phi_{12}(A)=U \cap \Phi_{12}(U \cap A)$.

Proof. It is similar to the proof of ([12], Theorem 2.10).

Note that if $\tau_{12}$ is a supra topology, then Theorem 4.2 above may be not satisfied as in Example 3.1, let $U=\{a, c\} \in \tau_{12}$ and take $A=\{a, b\}$. Then $\Phi_{12}(\{a, b\})=X, U \cap A=\{a\}$ and $\Phi_{12}(U \cap A)=\{a\}$, then $U \cap \Phi_{12}(A)=\{a, c\}$ while, $U \cap \Phi_{12}(U \cap A)=\{a\} \neq U \cap \Phi_{12}(A)$.

Theorem 4.3. If $\mathcal{G}$ is a grill on a bts $\left(X, \tau_{1}, \tau_{2}\right)$ such that $\tau_{12}$ is a topology and $\tau_{12} \backslash\{\phi\} \subseteq \mathcal{G}$. Then for all $U \in \tau_{12}, U \subseteq \Phi_{12}(U)$.

\section{Proof.}

In case $U=\emptyset$, we obviously have $\Phi_{12}(U)=\phi$. Now, if $\tau_{12} \backslash\{\emptyset\} \subseteq \mathcal{G}$, then $\Phi_{12}(X)=X$. In fact, $x \notin \Phi_{12}(X) \Rightarrow \exists V \in \tau_{12}(x)$ such that $V \cap X \notin \mathcal{G} \Rightarrow$ $V \notin \mathcal{G}$ a contradiction. Now, by the above theorem, for any $U \in \tau_{12} \backslash\{\emptyset\}$ we have, $U \cap \Phi_{12}(X)=U \cap \Phi_{12}(U \cap X)$

$\Rightarrow U \cap X=U \cap \Phi_{12}(U)$

$\Rightarrow U=U \cap \Phi_{12}(U)$

$\Rightarrow U \subseteq \Phi_{12}(U)$. 
The following example shows that if $\tau_{12}$ is not a topology on $X$, then the above Theorem does not satisfied.

Example 4.2. Let $X=\{a, b, c\}, \tau_{1}$ and $\tau_{2}$ be two topologies on $X$ such that $\tau_{1}=\{\emptyset, X,\{a, c\}\}$ and $\tau_{2}=\{\emptyset, X,\{b, c\}\}$. Let $\mathcal{G}=\{\{a\},\{a, b\}\{a, c\}, X\}$, $U=\{b, c\}$. Then $\tau_{12}=\{\emptyset, X,\{a, c\},\{b, c\}\}$. Hence $\Phi_{12}(U)=\Phi_{12}(\{b, c\})=\emptyset$ and consequently $\{b, c\} \nsubseteq \Phi_{12}(\{b, c\})$.

Lemma 4.1. For any grill $\mathcal{G}$ on a bts $\left(X, \tau_{1}, \tau_{2}\right)$ and $A, B \subseteq X$ such that $\Phi_{12}(A \cup B)=\Phi_{12}(A) \cup \Phi_{12}(B)$, we have $\Phi_{12}(A) \backslash \Phi_{12}(B)=\Phi_{12}(A \backslash B) \backslash \Phi_{12}(B)$.

Proof. It is similar to the proof of Lemma 2.12 in [12].

Note that if $\tau_{12}$ is a supra topology, then $\Phi_{12}(A \cup B) \neq \Phi_{12}(A) \cup \Phi_{12}(B)$ and consequently Lemma 4.1 is not satisfied as in the following example.

Example 4.3. Let $X=\{a, b, c\}, \tau_{1}$ and $\tau_{2}$ be two topologies on $X$ such that $\tau_{1}=\{\emptyset, X,\{a, c\}\}$ and $\tau_{2}=\{\emptyset, X,\{b, c\}\}$. Let $\mathcal{G}=P(X) \backslash\{\emptyset\}, A=\{a\}$, $B=\{b\}$. Then, $\tau_{12}=\{X, \emptyset,\{a, c\},\{b, c\}\}$,

$\Phi_{12}(A)=\{a\}, \Phi_{12}(B)=\{b\} . \quad$ Also, $\Phi_{12}(A \cup B)=\Phi_{12}(\{a, b\})=X \neq$ $\Phi_{12}(A) \cup \Phi_{12}(B)=\{a, b\}$. Also, take $A=\{a, b\}, B=\{b\}$. Then $\Phi_{12}(A)=$ $\Phi_{12}(\{a, b\})=X, \Phi_{12}(B)=\{b\}$ and $A \backslash B=\{a\} \Rightarrow \Phi_{12}(A \backslash B)=\{a\}$. Hence, $\Phi_{12}(A) \backslash \Phi_{12}(B)=\{a, c\} \neq \Phi_{12}(A \backslash B) \backslash \Phi_{12}(B)=\{a\}$.

Corollary 4.2. Let $\mathcal{G}$ be a grill on a space $\left(X, \tau_{1}, \tau_{2}\right)$ such that $\Phi_{12}(A \cup B)=$ $\Phi_{12}(A) \cup \Phi_{12}(B)$ and $B \notin \mathcal{G}$. Then $\Phi_{12}(A \cup B)=\Phi_{12}(A)=\Phi_{12}(A \backslash B)$

\section{Proof.}

$\Phi_{12}(A \cup B)=\Phi_{12}(A) \cup \Phi_{12}(B)$ (by Proposition $\left.3.1(\mathrm{iii})\right)=\Phi_{12}(A)$.

Also, $\Phi_{12}(A \backslash B) \subseteq \Phi_{12}(A)$ (by Proposition 3.1 (i)). From the above lemma, $\Phi_{12}(A) \backslash \Phi_{12}(B)=\Phi_{12}(A \backslash B) \backslash \Phi_{12}(B) \Rightarrow \Phi_{12}(A)=\Phi_{12}(A \backslash B)$.

Finally, we have $\Phi_{12}(A \cup B)=\Phi_{12}(A)=\Phi_{12}(A \backslash B)$.

Note that, if $\left(X ; \tau_{12}\right)$ is a supra topological space, then $\Phi_{12}(A \cup B) \neq$ $\Phi_{12}(A) \cup \Phi_{12}(B)$, but the converse is not true in general as in the following example.

Example 4.4. Let $X=\{a, b, c\}, \tau_{1}=\{X, \emptyset,\{a, b\}\}, \tau_{2}=\{X, \emptyset,\{a, c\}\}$. Then, $\tau_{12}=\{X, \emptyset,\{a, b\},\{a, c\}\}$. Let $\mathcal{G}=\{X,\{a\},\{a, b\},\{a, c\}\}$. Then $\Phi_{12}(A \cup B)=$ $\Phi_{12}(A) \cup \Phi_{12}(B), \forall A, B \subseteq X$, although $\tau_{12}$ is a supra topology.

Theorem 4.4. Let $\mathcal{G}$ be a grill on a space $\left(X, \tau_{1}, \tau_{2}\right)$ such that $A \subseteq \Phi_{12}(A)$, then $\tau^{*}{ }_{12}-c l(A) \subseteq c l_{12}(A)=\operatorname{cl}_{12}\left(\Phi_{12}(A)\right)=\Phi_{12}(A)$. 


\section{Proof.}

Since $\tau^{*}{ }_{12}$ is finer than $\tau_{12}$, then $c l^{*}{ }_{12}(A) \subseteq c l_{12}(A)$. Also, $c l_{12}\left(\Phi_{12}(A)\right)=$ $\Phi_{12}(A) \subseteq l_{12}(A)$ (by Proposition 3.2 (ii)).

Further, $A \subseteq \Phi_{12}(A) \Rightarrow c l_{12}(A) \subseteq c_{12}\left(\Phi_{12}(A)\right) \Rightarrow c l_{12}(A) \subseteq \Phi_{12}(A)$ Hence, $c l^{*}{ }_{12}(A) \subseteq c l_{12}(A)=\Phi_{12}(A)=\operatorname{cl}_{12}\left(\Phi_{12}(A)\right)$.

Note that, if $\tau_{12}$ is a topology, then $\tau_{12}^{*}-c l(A)=c l_{12}(A)=c_{12}\left(\Phi_{12}(A)\right)=$ $\Phi_{12}(A)$.

Now, we introduce the notion of a semi P.open subsets of a space $\left(X, \tau_{1}, \tau_{2}\right)$.

Definition 4.2. Let $\left(X, \tau_{1}, \tau_{2}\right)$ be a bts and $A \subseteq X$. Then $A$ is said to be semi P.open if $\exists$ a P.open set $G$ such that $G \subseteq A \subseteq c_{12}(G)$. The complement of semi P.open is a semi P-closed.

Note that every P.open (resp. P.closed) set is semi P.open (P.closed) set.

Corollary 4.3. Let $\mathcal{G}$ be a grill on a bts $\left(X, \tau_{1}, \tau_{2}\right)$ and $\tau_{12}$ is a topology on $X$. Then the following are equivalent:-

1. For all P.open in $\left(X ; \tau_{1} ; \tau_{2}\right), A \subseteq \Phi_{12}(A)$

2. For any semi P.open set $A$ in $X, A \subseteq \Phi_{12}(A)$

\section{Proof.}

$(2) \Rightarrow(1)$ is obvious, since every P.open is semi P.open.

$(1) \Rightarrow(2)$ Let $A$ be a semi P.open in $X$. Then $\exists$ P.open set $G$ in $\left(X ; \tau_{1} ; \tau_{2}\right)$ such that $G \subseteq A \subseteq c l_{12}(G)$. Since $G \in \tau_{12}$, by (1), $G \subseteq \Phi_{12}(G)$. Then by Theorem (4.4) $A \subseteq c_{12}(G)=\Phi_{12}(G) \subseteq \Phi_{12}(A) \Rightarrow A \subseteq \Phi_{12}(A)$.

\section{$5 \quad$ Supra toplogy suitable for a grill}

In this section we shall consider grills satisfying a certain condition and this will make the grills suitable and compatible vis-a-vis the topology of the space.

Definition 5.1. Let $\mathcal{G}$ be a grill on a bts $\left(X, \tau_{1}, \tau_{2}\right)$. Then $\tau_{12}$ is said to be suitable for $\mathcal{G}$ if $\forall A \subseteq X, A \backslash \Phi_{12}(A) \notin \mathcal{G}$. 
We now give some characterization of the notion of the suitable.

Theorem 5.1. For a grill $\mathcal{G}$ on a bts $\left(X, \tau_{1}, \tau_{2}\right)$, the following are equivalent:-

1. $\tau_{12}$ is suitable for $\mathcal{G}$

2. For any P.closed subset $A$ in $\left(X, \tau_{1}^{*}, \tau_{2}^{*}\right)$ (i.e $A$ is $\tau_{12}^{*}$-closed), $A \backslash \Phi_{12}(A) \notin$ $\mathcal{G}$.

3. $A \subseteq X$ and $A \cap \Phi_{12}(A)=\emptyset \Rightarrow A \notin \mathcal{G}$.

\section{Proof.}

$(1) \Rightarrow(2)$ Obviously by the definition.

$(2) \Rightarrow(3)$ Let $(2)$ holds and let $A \subseteq X$ and $A \cap \Phi_{12}(A)=\emptyset$ such that $A \in \mathcal{G}$. Then $A=A \backslash \Phi_{12}(A) \in \mathcal{G}$. By $(2), A$ is not P.closed in $\left(X, \tau_{1}^{*} ; \tau_{2}^{*}\right)$. So, $\Phi_{12}(A) \nsubseteq A$ and this implies $\Phi_{12}(A) \cap A \neq \emptyset$ which a contradiction, otherwise, $A \cap \Phi_{12}(A)=\emptyset \Rightarrow A=\emptyset$ or $\Phi_{12}(A)=\emptyset$. In both cases we have $\emptyset \nsubseteq A$ which a contradiction. Hence $A \notin \mathcal{G}$.

$(3) \Rightarrow(1)$ Let $\forall A \subseteq X$ and $A \cap \Phi_{12}(A)=\emptyset \Rightarrow A \notin \mathcal{G}$. We first claim that $A \backslash \Phi_{12}(A) \cap \Phi_{12}\left(A \backslash \Phi_{12}(A)\right)=\emptyset$. In fact, $x \in A \backslash \Phi_{12}(A) \Rightarrow x \in A$ and $x \notin \Phi_{12}(A) \Rightarrow \exists O_{x} \in \tau_{12}(x)$ such that $O_{x} \cap A \notin \mathcal{G}$. Now $O_{x} \cap\left(A \backslash \Phi_{12}(A)\right) \subseteq$ $O_{x} \cap A \notin \mathcal{G} \Rightarrow x \notin \Phi_{12}\left(A \backslash \Phi_{12}(A)\right)$. Hence $\left(A \backslash \Phi_{12}(A)\right) \cap \Phi_{12}\left(A \backslash \Phi_{12}(A)\right)=\emptyset$. By (3), $A \backslash \Phi_{12}(A) \notin \mathcal{G} \forall A \subseteq X$. Hence the result.

Theorem 5.2. Let $\mathcal{G}$ be a grill on a bts $\left(X ; \tau_{1} ; \tau_{2}\right)$ and $\Phi_{12}(A \cup B)=\Phi_{12}(A) \cup$ $\Phi_{12}(B)$. The following are equivalent and each is a necessary condition for $\tau_{12}$ to be suitable for the grill $\mathcal{G}$.

1. For any $A \subseteq X, A \cap \Phi_{12}(A)=\emptyset \Rightarrow \Phi_{12}(A)=\emptyset$.

2. For any $A \subseteq X, \Phi_{12}\left(A \backslash \Phi_{12}(A)\right)=\emptyset$

3. For any $A \subseteq X, \Phi_{12}\left(A \cap \Phi_{12}(A)\right)=\Phi_{12}(A)$

\section{Proof.}

$(1) \Rightarrow(2)$ It follows by noting that $\left(A \backslash \Phi_{12}(A)\right) \cap \Phi_{12}\left(A \backslash \Phi_{12}(A)\right)=\emptyset, \forall A \subseteq$ $X$.(see Theorem 5.1)

$(2) \Rightarrow(3) A=\left(A \backslash\left(A \cap \Phi_{12}(A)\right)\right) \cup\left(A \cap \Phi_{12}(A)\right)$

$\Rightarrow \Phi_{12}(A)=\Phi_{12}\left(A \backslash\left(A \cap \Phi_{12}(A)\right)\right) \cup \Phi_{12}\left(A \cap \Phi_{12}(A)\right)$

$\Rightarrow \Phi_{12}(A)=\Phi_{12}\left(A \backslash \Phi_{12}(A)\right) \cup \Phi_{12}\left(A \cap \Phi_{12}(A)\right)$

$\Rightarrow \Phi_{12}(A)=\Phi_{12}\left(A \cap \Phi_{12}(A)\right)(b y(2))$.

$(3) \Rightarrow(1)$ Let $A \subseteq X$ and $A \cap \Phi_{12}(A)=\emptyset$. Then, $\Phi_{12}\left(A \cap \Phi_{12}(A)\right)=\Phi_{12}(A) \Rightarrow$ $\Phi_{12}(A)=\emptyset$.

Corollary 5.1. If $\tau_{12}$ is suitable for a grill $\mathcal{G}$ and $\Phi_{12}(A \cup B)=\Phi_{12}(A) \cup$ $\Phi_{12}(B)$, then $\Phi_{12}$ is an idempotent, i.e $\Phi_{12}\left(\Phi_{12}(A)\right)=\Phi_{12}(A), \forall A \subseteq X$. 


\section{Proof.}

By Theorem 5.2 and Proposition 3.1(i), we get, $\Phi_{12}(A)=\Phi_{12}\left(A \cap \Phi_{12}(A)\right) \subseteq$ $\Phi_{12}(A) \cap \Phi_{12}\left(\Phi_{12}(A)\right)=\Phi_{12}\left(\Phi_{12}(A)\right)$ by Proposition $3.2\left(\right.$ ii). Hence $\Phi_{12}\left(\Phi_{12}(A)\right)=$ $\Phi_{12}(A)$.

Theorem 5.3. Let $\mathcal{G}$ be a grill on a bts $\left(X, \tau_{1}, \tau_{2}\right)$ such that $\tau_{12}$ is suitable for the grill $\mathcal{G}$. Also, let $\Phi_{12}(A \cup B)=\Phi_{12}(A) \cup \Phi_{12}(B)$. Then $A \subseteq X$ is P.closed in $\left(X ; \tau_{1}^{*} ; \tau_{2}^{*}\right)$ iff it can be expressed as a union of a set which is P.closed in $\left(X, \tau_{1}, \tau_{2}\right)$ and a set not in $\mathcal{G}$.

\section{Proof.}

Let $A$ be a P.closed subset in $\left(X, \tau_{1}^{*}, \tau_{2}^{*}\right)$, then $\Phi_{12}(A) \subseteq A$. Now, $A=\Phi_{12}(A) \cup$ $A \backslash \Phi_{12}(A)$. Since $\tau_{12}$ is suitable for $\mathcal{G}, A \backslash \Phi_{12}(A) \notin \mathcal{G}$ and by Proposition 3.2(ii) $\Phi_{12}(A)$ is P.closed in $\left(X ; \tau_{1} ; \tau_{2}\right)$.

Conversely, let $A=F \cup B$, where $F$ is P.closed in $\left(X, \tau_{1}, \tau_{2}\right)$ (i.e $\tau_{12}$-closed) and $B \notin \mathcal{G}$. Then $\Phi_{12}(A)=\Phi_{12}(F) \cup \Phi_{12}(B)=\Phi_{12}(F)$ (by Propositions 3.1 and $3.2) \subseteq c_{12}(F)=F \subseteq A$. Hence $\Phi_{12}(A) \subseteq A$ and consequently $A$ is P.closed in $\left(X, \tau_{1}^{*}, \tau_{2}^{*}\right)$.

Corollary 5.2. Let $\left(X, \tau_{1}, \tau_{2}\right)$ be a bts and $\mathcal{G}$ be a grill on $X$. Also, let $\tau_{12}$ be a suitable for $\mathcal{G}$. Then $\beta\left(\mathcal{G}, \tau_{12}\right)$ is a supra topology on $X$ and $\beta\left(\mathcal{G}, \tau_{12}\right)=\tau_{12}^{*}$.

\section{Proof.}

Let $U$ be a P.open in $\left(X, \tau_{1}^{*}, \tau_{2}^{*}\right)\left(\right.$ or $\left.U \in \tau_{12}^{*}\right)$. Then by Theorem 5.3, $X \backslash U=$ $F \cup B$ where $F$ is $\tau_{12}$-closed and $B \notin \mathcal{G}$. Then, $U=X \backslash F \cap X \backslash B \Rightarrow U=$ $(X \backslash F) \backslash B)=V \backslash B$, where $V \in \tau_{12}$ and $B \notin \mathcal{G}$. Thus every $\tau_{12}^{*}$-open is of the form $V \backslash B$. Hence $\beta\left(\mathcal{G}, \tau_{12}\right)=\tau_{12}^{*}$.

Theorem 5.4. Let $\mathcal{G}$ be a grill on a bts $\left(X, \tau_{1}, \tau_{2}\right)$ such that $\tau_{12} \backslash\{\emptyset\} \subseteq \mathcal{G}$ and $\tau_{12}$ is a topology suitable for the grill $\mathcal{G}$. Let $G$ be P.open set in $\left(X, \tau_{1}^{*}, \tau_{2}^{*}\right)$ such that $G=U \backslash A$, where $U \in \tau_{12}$ and $A \notin \mathcal{G}$. Then, $c_{12}^{*}(G)=c_{12}(G)=$ $\Phi_{12}(G)=\Phi_{12}(U)=c l_{12}(U)=c l_{12}^{*}(U)$.

\section{Proof.}

Let $G=U \backslash A$, where $U \in \tau_{12}$ and $A \notin \mathcal{G}$ (note that in view of Corollary 5.2, Every $\tau_{12}^{*}$-open set is of this form). Since $\tau_{12} \backslash\{\emptyset\} \subseteq \mathcal{G}$ by Theorem 4.3 , we have $U \subseteq \Phi_{12}(U)$. Hence by Theorem 4.4,

$$
\Phi_{12}(U)=c l_{12}(U)=c l_{12}^{*}(U)
$$

Now, $G$ being $\tau_{1}^{*} 2$-open set, we claim that $G \subseteq \Phi_{12}(G)$. In fact, $c l_{12}^{*}(X \backslash G)=$ $X \backslash G \Rightarrow \Phi_{12}(X \backslash G) \subseteq X \backslash G \Rightarrow \Phi_{12}(X) \backslash \Phi_{12}(G)=\Phi_{12}(X \backslash G) \backslash \Phi_{12}(G) \subseteq$ $\Phi_{12}(X \backslash G) \subseteq X \backslash G \Rightarrow \Phi_{12}(X) \backslash \Phi_{12}(G) \subseteq X \backslash G$. (by Lemma 4.1) $\Rightarrow$ $X \backslash \Phi_{12}(G) \subseteq X \backslash G \Rightarrow G \subseteq \Phi_{12}(G)$. Hence, by Theorem 4.4

$$
c l_{12}(G)=c l_{12}^{*}(G)=\Phi_{12}(G)
$$


Again, $G \subseteq U \Rightarrow \Phi_{12}(G) \subseteq \Phi_{12}(U)$ and also, $\Phi_{12}(G)=\Phi_{12}(U \backslash A) \supseteq$ $\Phi_{12}(U) \backslash \Phi_{12}(A) \supseteq \Phi_{12}(U)$. ( by Lemma 4.1$)=\Phi_{12}(U)($ as $A \notin \mathcal{G})$. So $\Phi_{12}(\mathcal{G})=$ $\Phi_{12}(U)$ and consequently,

$$
\Phi_{12}(G)=\Phi_{12}(U)
$$

From 1,2 and 3 we have the required result.

Theorem 5.5. Let $\mathcal{G}$ be a grill on a bts $\left(X ; \tau_{1} ; \tau_{2}\right)$ such that $\tau_{12}$ is a topology suitable for the grill $\mathcal{G}$. Then $\forall G \in \tau_{12}$ and $A \subseteq X, \Phi_{12}(G \cap A)=\Phi_{12}(G \cap$ $\left.\Phi_{12}(A)\right)=c_{12}\left(G \cap \Phi_{12}(A)\right)$

Proof. It is similar to the proof of Theorem 3.8 in [12].

Corollary 5.3. Let $\mathcal{G}$ be a grill on a bts $\left(X, \tau_{1}, \tau_{2}\right)$ such that $\tau_{12}$ is a topology suitable for the grill $\mathcal{G}$. If $G \in \tau_{12} \backslash \mathcal{G}$, then $G \subseteq X \backslash \Phi_{12}(X)$.

\section{Proof.}

Taking $A=X$ in Theorem 5.5, we get, $c l_{12}\left(G \cap \Phi_{12}(X)\right)=\Phi_{12}(G \cap X)=$ $\Phi_{12}(G) \forall G \in \tau_{12}$. Now, if $G \notin \mathcal{G}$, then $\Phi_{12}(G)=\emptyset$ and also, $c l_{12}\left(G \cap \Phi_{12}(X)\right)=$ $\emptyset \Rightarrow \Phi_{12}\left(G \cap \Phi_{12}(X)\right)=\emptyset \Rightarrow G \subseteq X \backslash \Phi_{12}(X)$.

Remark 5.1. For any grill $\mathcal{G}$ on a bts $\left(X, \tau_{1}, \tau_{2}\right)$. Let $A^{d^{*}}$ and $A^{d}$ denote to the drived set of $A$ with respect to $\tau_{12}^{*}$ and $\tau_{12}$ respectively. Then

1. $A_{12}^{d^{*}} \subseteq A_{12}^{d}$ and

2. $A_{12}^{d^{*}} \subseteq \Phi_{12}(A)$.

In fact, (1) follows from the fact $\tau_{12} \subseteq \tau_{12}^{*}$ and for (2) we have $x \in A_{12}^{d^{*}} \Rightarrow \in$ $c l_{12}^{*}(A \backslash\{x\})=A \backslash \backslash\{x\} \cup \Phi_{12}(A\{x\}) \Rightarrow x \in \Phi_{12}(A \backslash\{x\}) \subseteq \Phi_{12}(A)$, i.e $A_{12}^{d^{*}} \subseteq \Phi_{12}(A)$.

Also, we have

Lemma 5.1. Let $\mathcal{G}$ be a grill on a bts $\left(X, \tau_{1}, \tau_{2}\right)$. Let $\Phi_{12}(A \cup B)=\Phi_{12}(A) \cup$ $\Phi_{12}(B)$ for some $x \in X,\{x\} \notin \mathcal{G}$. Then, $x \in \Phi_{12}(A) \Leftrightarrow x \in A_{12}^{d^{*}}, \forall A \subseteq X$

\section{Proof.}

Let $A \subseteq X$. Then $A_{12}^{d^{*}} \subseteq \Phi_{12}(A)$ follows from Remark 5.1. Now, suppose that $\{x\} \notin \mathcal{G}$. Then $x \in \Phi_{12}(A) \Rightarrow x \in \Phi_{12}(A \backslash\{x\})$ (by Corollary 4.2) $\Rightarrow x \in c l_{12}^{*}(A \backslash\{x\}) \Rightarrow x \in A_{12}^{d^{*}}$

Definition 5.2. [11] A grill $\mathcal{G}$ on $X$ is said to be a $\sigma$-grill if for any countable collection $\left\{A_{n}: n \in \mathbb{N}\right\}$ of subsets of $X, \cup_{n=1}^{\alpha} A_{n} \notin \mathcal{G}$ whenever $A_{n} \notin \mathcal{G} \forall n \in \mathbb{N}$

Theorem 5.6. Let $\mathcal{G}$ be a $\sigma$-grill on a hereditarily Lindelöf space $\left(X ; \tau_{1} ; \tau_{2}\right)$, then $\tau_{12}$ is a suitable for the grill $\mathcal{G}$. 


\section{Proof.}

Let $A \subseteq X$ such that $A \cap \Phi_{12}(A)=\emptyset$. Then $\forall x \in A \exists O_{x} \in \tau_{12}(x)$ such that $O_{x} \cap A \notin \mathcal{G}$. Now, $\left\{O_{x} \cap A: x \in A\right\}$ is a $\tau_{12 A^{-}}$open cover of $A$, by Lindelöfness of $A$, there exists a countable subset $\left\{x_{n}: n \in \mathbb{N}\right\}$ of $A$ such that $A=\cup_{i=1}^{\infty}\left(U_{x_{i}} \cap A\right)$. As $\mathcal{G}$ is a $\sigma$-grill it follows that $A \notin \mathcal{G}$ and hence by Theorem 5.1, $\tau_{12}$ becomes suitable for $\mathcal{G}$.

Theorem 5.7. Let $\mathcal{G}$ be a grill on a space $\left(X, \tau_{1}, \tau_{2}\right)$ such that $\tau_{12}$ is a suitable for the grill $\mathcal{G}$ and for each $x \in X,\{x\} \notin \mathcal{G}$. Also, let $\Phi_{12}(A \cup B)=\Phi_{12}(A) \cup$ $\Phi_{12}(B) \forall A, B \subseteq X$. If $A \subseteq X$ is P.closed in $\left(X ; \tau_{1}^{*} ; \tau_{2}^{*}\right)$, then $A$ can be written as a union of a P.perfect set in $\left(X, \tau_{1}, \tau_{2}\right)$ and a set $\notin \mathcal{G}$.

\section{Proof.}

Let $A \subseteq X$ be P.closed in $\left(X, \tau_{1}^{*}, \tau_{2}^{*}\right)$. Then by Theorem 5.3, $A=\Phi_{12}(A) \cup B$, where $\Phi_{12}(A)$ is P.closed in $\left(X, \tau_{1}, \tau_{2}\right)$ and $B \notin \mathcal{G}$. Since $\tau_{12}$ is suitable for $\mathcal{G}$, by Corollary 5.1, $\Phi_{12}(A)=\Phi_{12}\left(\Phi_{12}(A)\right)$. Now, $\Phi_{12}(A)$ being $\tau_{12}$-closed,

$$
\left(\Phi_{12}(A)\right)_{12}^{d} \subseteq \Phi_{12}(A)
$$

Again as $\{x\} \notin \mathcal{G} \forall x \in X, \Phi_{12}(B) \subseteq B_{12}^{d^{*}} \forall B \subseteq X$ ( Using Lemma 5.1). So $\Phi_{12}\left(\Phi_{12}(A)\right) \subseteq\left(\Phi_{12}(A)\right)_{12}^{d^{*}} \subseteq\left(\Phi_{12}(A)\right)_{12}^{d}$. So

$$
\Phi_{12}(A) \subseteq\left(\Phi_{12}(A)\right)_{12}^{d}
$$

from 4 and $5, \Phi_{12}(A)=\left(\Phi_{12}(A)\right)_{12}^{d}$, Showing that $\Phi_{12}(A)$ is a P.perfect in $\left(X, \tau_{1}, \tau_{2}\right)$.

We now take up a few topological properties, specially certain separation axioms, and study them briefly in respect of the families $\tau_{12}$ and $\tau_{12}^{*}$. The first part of the following theorem is similar to the first part of Theorem 3.18 in [12] and we shall prove the second part. At the first we introduce the following definitions which are found in [7].

Definition 5.3. Let $\left(X, \tau_{1}, \tau_{2}\right)$ be a bts. Then it is called:

1. $P^{*} T_{2}$ (or $P^{*}$-Hausdorff) if $\forall x, y \in X, x \neq y, \exists$ P.open sets $G, H$ such that $x \in G, y \in H$ and $G \cap H=\emptyset$

2. $P^{*}$-Urysohn or $P^{*} T_{2 \frac{1}{2}}$ if $\forall x, y \in X, x \neq y, \exists P$.open sets $G, H$ such that $x \in G, y \in H$ and $c l_{12}(G) \cap c_{12}(H)=\emptyset$

3. $P^{*}$-regular if $\forall x \in X, \forall P$.open set $G$ such that $x \in G, \exists P$.open sets $H$ such that $x \in H \subseteq \operatorname{cl}_{12}(H) \subseteq G$.

Theorem 5.8. Let $\mathcal{G}$ be a grill on a space $\left(X, \tau_{1}, \tau_{2}\right)$ such that $\tau_{12}$ a topology on $X$, suitable for the grill $\mathcal{G}$ and $\tau_{12} \backslash\{\emptyset\} \subseteq \mathcal{G}$. Then 
1. $\left(X, \tau_{1}, \tau_{2}\right)$ is $P^{*} T_{2}$ or $P^{*}$-Urysohn iff $\left(X, \tau_{1}^{*}, \tau_{2}^{*}\right)$ is respectively so.

2. If $\left(X, \tau_{1}^{*}, \tau_{2}^{*}\right)$ is $P^{*}$-regular, then $\tau_{12}=\tau_{12}^{*}$

Proof.As mentioned, we shall prove part (2) and for part (1) see [12]. so, for any $A \subseteq X$, we clearly have $c l_{12}^{*}(A) \subseteq c l_{12}(A)$ since $\tau_{12} \subseteq \tau_{12}^{*}$. Now, let $x \notin c l_{12}^{*}(A)$. Then for some P.open set $G$ in $\left(X, \tau_{1}^{*}, \tau_{2}^{*}\right)$ containing $x$ we have $G \cap A=\emptyset$. By $P^{*}$-regularity of $\left(X, \tau_{1}^{*}, \tau_{2}^{*}\right), \exists$ P.open set $H$ in $\left(X, \tau_{1}^{*}, \tau_{2}^{*}\right)$ such that $H=U \backslash B$, where $U \in \tau_{12}$ and $B \notin \mathcal{G}$ and also, $x \in H \subseteq c l_{12}^{*}(H) \subseteq G$.

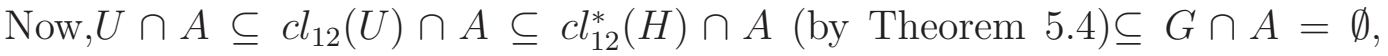
where $x \in U \in \tau_{12} \Rightarrow x \notin c l_{12}(A)$. Hence $c l_{12}(A) \subseteq c l_{12}^{*}(A)$ and consequently, $c l_{12}(A)=c l_{12}^{*}(A) \forall A \subseteq X$, which proves that $\tau_{12}=\tau_{12}^{*}$

Remark 5.2. It follows from the above theorem that if $\mathcal{G}$ is a grill on $X$ such that $\tau_{12} \backslash\{\emptyset\} \subseteq \mathcal{G}$ and $\tau_{12}$ is a suitable for the grill $\mathcal{G}$, then $\left(X, \tau_{1}, \tau_{2}\right)$ is $P^{*}$ regular whenever $\left(X, \tau_{1}^{*}, \tau_{2}^{*}\right)$ is $P^{*}$-regular. But the converse of this theorem may fail as is shown in the following example.

Example 5.1. Let $X=\{a, b, c\}, \tau_{1}=\{X, \emptyset,\{a\}\}$ and $\tau_{2}=\{X, \emptyset,\{b, c\}\}$. Then $\left(X ; \tau_{1} ; \tau_{2}\right)$ is $P^{*}$-regular. If $\mathcal{G}=\{\{a\},\{b\},\{a, b\},\{a, c\},\{b, c\}, X\}$. Then $\mathcal{G}$ is a grill on $X$ such that $\tau_{12} \backslash\{\emptyset\} \subseteq \mathcal{G}$. Also, $A \subseteq X, A \cap \Phi_{12}(A)=$ $\emptyset \Rightarrow A \notin \mathcal{G}$, i.e $\tau_{12}$ is a suitable for the grill $\mathcal{G}$. Also, we have $\tau_{12}^{*}=$ $\{X, \emptyset,\{a\},\{b, c\},\{b\},\{a, b\}\}$. Hence $\left(X, \tau_{12}^{*}\right)$ or $\left(X, \tau_{1}^{*}, \tau_{2}^{*}\right)$ is not $P^{*}$-regular. Since, $b \notin\{a, c\},\{a, c\}$ is P.closed in $\left(X ; \tau_{1}^{*} ; \tau_{2}^{*}\right)$ and all P.open sets containing $b$ intersect $X$ which is the only P.open set containing $\{a, c\}$

Our next example shows that under the stated conditions of Theorem 5.8 $\tau_{12}$ may coincide with $\tau_{12}^{*}$ even $\left(X, \tau_{1}^{*}, \tau_{2}^{*}\right)$ is not $P^{*}$-regular.

Example 5.2. Let $X$ be an uncountable set and $\left(X, \tau_{1}, \tau_{2}\right)$ be a bts such that and $\tau_{1}$ is the indiscrete topology and $\tau_{2}=\tau_{c o}$, the co-countable topology. Let $\mathcal{G}$ be the grill of all uncountable subsets of $X$. Then $\tau_{12}=\tau_{c o}$ and $\left(X, \tau_{12}\right)$ is a hereditarily Lindelöf space and $\mathcal{G}$ is $\sigma$-grill and by the Theorem $5.6 \tau_{12}$ is a suitable for the grill $\mathcal{G}$. Also, clearly, $\tau_{12} \backslash\{\emptyset\} \subseteq \mathcal{G}$. We show that $\tau_{12}=\tau_{c o}=\tau_{12}^{*}$ or $\tau_{c o}=\tau_{c o}^{*}$. Indeed, for $V \in \tau_{c o}^{*}$ with $V=U \backslash A$, where $U \in \tau_{c o}$ and $A \notin \mathcal{G}$. $U^{\prime}$ and $A$ are countable. Now, $V^{\prime}=X \cap\left(U \cap A^{\prime}\right)^{\prime}=X \cap\left(U^{\prime} \cup A\right)=U^{\prime} \cup A$, which is countable. Then $V \in \tau_{c o}$. It follows that $\tau_{c o}=\tau_{c o}^{*}$ and clearly $\left(X, \tau_{c o}\right)$ and $\left(X, \tau_{c o}^{*}\right)$ are not $P^{*}$-regular.

Theorem 5.9. Let $\mathcal{G}$ be a grill on a bts $\left(X, \tau_{1}, \tau_{2}\right)$ such that $\tau_{12} \backslash\{\emptyset\} \subseteq \mathcal{G}$ and that $\tau_{12}$ is a topology suitable for the grill $\mathcal{G}$. Then $\left(X, \tau_{1}, \tau_{2}\right)$ is $P^{*}$-connected $\Leftrightarrow\left(X, \tau_{1}^{*}, \tau_{2}^{*}\right)$ is $P^{*}$-connected

Proof. It is similar to the proof of Theorem 3.22 in [12]. 
The following example shows that if $\tau_{12}$ is a supra topology, then $\left(X ; \tau_{1} ; \tau_{2}\right)$ is connected while $\left(X ; \tau_{1}^{*} ; \tau_{2}^{*}\right)$ is not connected. In fact, Let $X=\{a, b, c\}, \tau_{1}=$ $\{X, \emptyset,\{a, b\}\}$ and $\tau_{2}=\{X, \emptyset,\{b, c\}\}$. Let $\mathcal{G}=P(X) \backslash\{\emptyset,\{b\}\}$. Then $\tau_{12}=$ $\{X, \emptyset,\{a, b\},\{b, c\}\}$ and $\left(X, \tau_{12}\right)$ is connected. Clearly, $\tau_{12} \backslash\{\emptyset\} \subseteq \mathcal{G}$ and $\forall A \subseteq X, A \cap \Phi_{12}(A)=\emptyset \Rightarrow A \notin \mathcal{G}$, i.e $\tau_{12}$ is a suitable for the grill $\mathcal{G}$. It is easy to see that:

$\tau_{12}^{*}=\{X, \emptyset,\{a\},\{c\},\{a, b\},\{b, c\},\{a, c\}\}$.

Hence $\tau_{12}^{*}$ is a supra topology and $\left(X, \tau_{12}^{*}\right)$ is not $P^{*}$ is connected, since $X=$ $\{a\} \cup\{b, c\}$

\section{References}

[1] M.E.Abd-El-Monsef and E.F Lashine, Some operation on supratopological spaces, Dirasat, XIII (7)( 1986), 43-50.

[2] K.C.Chattopadhyay and W. J. Thron, Extensions of closure spaces, can.J. Math., 29 (6)( 1977), 1277-1286.

[3] K.C.Chattopadhyay, O. Njastad and W. J. Thron, Metrotopic spaces and extension of closure spaces, Can.J. Math., 35 (4) (1983), 613-629.

[4] G.Choquet, Surles notions de finite et grille, Comples Rendus Acad, Sci Paris, 224 (1947), 171-173.

[5] B.P.Dvalish vili, Bitopological Spaces Theory, Relations with Generalized Algebraic Structure and Applications, Mathematics Studies, 2005.

[6] S.A.El-Sheikh, A new approach to fuzzy bts's, INS, 173( 2001), 283-301.

[7] A.Kandel, H.Fawaz and S.A.El-Sheikh, On bitopological spaces, Proc. Assuit First Inter. Confer. Part III (1990), 115-132.

[8] A.Kandel, A.Nouch and S.A.El-Sheikh, On fuzzy bitopological spaces, FSS, 74 (1995), 353-363.

[9] J.C.Kelley, Bitopological spaces, Proc. London Math. Soc., 13 (1963), $71-79$.

[10] K.Kuratowski, Topologi, Warsazawa, 1933.

[11] N.Levine, Semi-Open sets and semi-continuity in topological spaces, Amer. Math. Monthly, 70(1993), 36-41.

[12] B.Roy and M. N.Mukherjee, On typical topology induced by grill, Soochow J. of ATHEMTICS, 33(4)( 2007), 772-786. 
[13] L.A.Steen and J. R.Seebach , Counter examples in topology, Springer Verlag, New York, 1978.

[14] W.J.Thron, Proximity structures and grills, Math. Ann, 206(1973), $35-62$.

Received: November 11, 2013 